\title{
Design of Multi-Layer Optical Fibers with Ring Refractive Index to Reduce Dispersion and Increase Bandwidth in Broadband Optical Networks
}

\author{
Mahmood Seifouri \\ Shahid Rajaee Teacher Training \\ University, Faculty of ECE \\ Lavizan, Tehran, Iran \\ mahmood.seifouri@srttu.edu
}

\author{
Mohammad Mehdi Karkhanehchi \\ Razi University, \\ Faculty of Engineering \\ Kermanshah, Iran \\ mkarkhanehchi@razi.ac.ir
}

\author{
Sohran Rohani \\ Shahid Rajaee Teacher Training \\ University, Faculty of ECE \\ Lavizan, Tehran, Iran \\ Shoran_rohani@yahoo.com
}

\begin{abstract}
The main goal in this paper is to design single-mode optical fibers for DWDM networks, which are used today in rapid communications. These networks require low dispersion in a wide range of wavelengths. So, in this paper, multi-layer optical fibers with low dispersion value and flat dispersion slope in wavelength range of $\left(1.5-1.6^{\mu \mathrm{m})}\right.$ are designed, using optimization algorithms.
\end{abstract}

Keywords- single-mode fiber; dispersion; dispersion slope; optimization algorithm.

\section{INTRODUCTION}

Triple-clad single-mode fibers have gain significant attention because of the perfect transmission properties that can be achieved by adjusting the multi-parameters [1-3]. A tripleclad fiber was firstly studied by Cozens and Boucouvalas as an optical coupler for sensing [4]. Dispersion curves for a particular coaxial structure were theoretically obtained with the resonance technique [5] and later by the solution of the transcendental equation [6]. In this paper, previous work reported in [5] is extended and the study is focused on the chromatic dispersion coefficient and the chromatic dispersion slope, and the design of triple-clad optical fibers with very low chromatic dispersion value and flat chromatic dispersion slope. A type of large effective area fibers with ring index profiles is proposed.

The effects made by the geometric parameters and optical parameters on the waveguide dispersion and cutoff characteristics of low order modes have been analyzed in detail. The calculated results indicate that the waveguide dispersion during single-mode propagation can be controlled by changing the optical and geometrical parameters. This means that it is possible to a obtain dispersion-flattened fiber with large effective-area along a region of wavelengths in WII type single-mode fibers [7]. The dispersion is optimized using a
Differential Evolution (DE) algorithm. Any similar structure can be optimized and controlled with this design. In section 2, a theoretical method to model the fiber structure and to obtain dispersion and dispersion slope in triple-clad fibers is investigated.

\section{THEORETICAL MODELING OF FIBER STRUCTURE}

Refractive index for WII structure is presented in Figure 1. Refractive index of each layer is as follows:

$\left\{\begin{array}{l}n_{1}, \rightarrow 0<r<a, \\ n_{2}, \rightarrow a<r<b, \\ n_{3}, \rightarrow b<r<c, \\ n_{4}, \rightarrow c<r,\end{array}\right.$

where $r$ is the position vector of fiber radius.

Optical parameters are defined as:

$R_{1}=\frac{n_{1}-n_{3}}{n_{3}-n_{2}}$

$R_{2}=\frac{n_{2}-n_{4}}{n_{3}-n_{2}}$

$\Delta=\frac{n_{1}^{2}-n_{4}^{2}}{n_{4}^{2}} \approx \frac{n_{1}-n_{4}}{n_{4}}$, 
Geometric parameters are defined as follows [8]:

$$
P=\frac{b}{c}, Q=\frac{a}{c}
$$

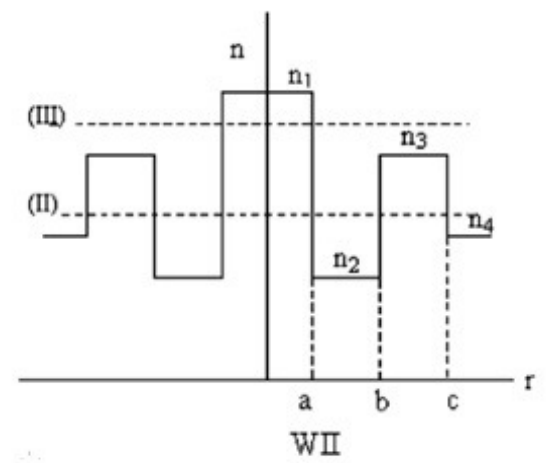

Fig. 1. refractive index structure in the WII fiber[8].

Another parameter considered as a geometric parameter is the fiber core radius $a$, in the first layer.

Other parameters are as follows:

$$
V=k_{0} a \sqrt{n_{1}^{2}-n_{4}^{2}}
$$

$B=\frac{\left(b / k_{0}\right)^{2}-n_{4}^{2}}{n_{1}^{2}-n_{4}^{2}}=1-\left(\frac{U_{1}}{V}\right)^{2}$,

where $\mathrm{V}$ is the normalized frequency, $\mathrm{B}$ is the normalized propagation constant [8] and $\mathrm{r}$ is the radial position. According to the effective refractive index given by $n_{\text {eff }}=\frac{\beta}{k_{0}}$ where $\beta$ is the longitudinal propagation constant of guided mode and $k_{0}$ is the wave number in free space, the structure of WII-type can be divided into two regions, which are shown in Figure 1. According to the wave equation, the boundary conditions of electromagnetic field and under the LP approximation, the characteristic equations in regions (II)-(III) can be as described in (14) and (15), and the parameters in these matrices are defined as follow:

$$
\begin{aligned}
& U_{1}=a \sqrt{k_{0}^{2} n_{1}^{2}-\beta^{2}}, \\
& U_{2}=b \sqrt{\beta^{2}-k_{0}^{2} n_{3}^{2}},
\end{aligned}
$$

$U_{3}=b \sqrt{k_{0}^{2} n_{3}^{2}-\beta^{2}}$

$W_{3}=a \sqrt{\beta^{2}-k_{0}^{2} n_{2}^{2}}$

$W_{4}=c \sqrt{\beta^{2}-k_{0}^{2} n_{4}^{2}}$

$W_{3}^{\prime}=\frac{P}{Q} W_{3}, U_{2}^{\prime}=\frac{1}{P} U_{2}, U_{3}^{\prime}=\frac{1}{P} U_{3}$,

$\left[\begin{array}{cccccc}J_{m}\left(U_{1}\right) & -I_{m}\left(W_{3}\right) & -K_{m}\left(W_{3}\right) & 0 & 0 & 0 \\ 0 & I_{m}\left(W_{3}^{\prime}\right) & K_{m}\left(W_{3}^{\prime}\right) & -J_{m}\left(U_{3}\right) & -Y_{m}\left(U_{3}\right) & 0 \\ 0 & 0 & 0 & J_{m}\left(U_{3}\right) & Y_{m}\left(U_{3}\right) & -K_{m}\left(W_{4}\right) \\ U_{1} J_{m}^{\prime}\left(U_{1}\right) & -W_{3} I_{m}^{\prime}\left(W_{3}\right) & -W_{3} K_{m}^{\prime}\left(W_{3}\right) & 0 & 0 & 0 \\ 0 & W_{3}^{\prime} I_{m}^{\prime}\left(W_{3}^{\prime}\right) & W_{3}^{\prime} K_{m}^{\prime}\left(W_{3}^{\prime}\right) & -U_{3} J_{m}^{\prime}\left(U_{3}\right) & -U_{3} Y_{m}^{\prime}\left(U_{3}\right) & 0 \\ 0 & 0 & 0 & U_{3}^{\prime} J_{m}^{\prime}\left(U_{3}^{\prime}\right) & U_{3}^{\prime} Y_{m}^{\prime}\left(U_{3}^{\prime}\right)-W_{4} K_{m}^{\prime}\left(W_{4}\right)\end{array}\right]$

$$
\left(n_{4}<n_{\text {eff }}<n_{3}, W I I\right),
$$




$$
\begin{aligned}
& {\left[\begin{array}{cccccc}
J_{m}\left(U_{1}\right) & -I_{m}\left(W_{3}\right) & -K_{m}\left(W_{3}\right) & 0 & 0 & 0 \\
0 & I_{m}\left(W_{3}^{\prime}\right) & K_{m}\left(W_{3}^{\prime}\right) & -I_{m}\left(U_{2}\right) & -K_{m}\left(U_{2}\right) & 0 \\
0 & 0 & 0 & I_{m}\left(U_{2}^{\prime}\right) & K_{m}\left(U_{2}^{\prime}\right)-K_{m}\left(W_{4}\right) \\
U_{1} J_{m}^{\prime}\left(U_{1}\right) & -W_{3} I_{m}^{\prime}\left(W_{3}\right) & -W_{3} K_{m}^{\prime}\left(W_{3}\right) & 0 & 0 & 0 \\
0 & W_{3}^{\prime} I_{m}^{\prime}\left(W_{3}^{\prime}\right) & W_{3}^{\prime} K_{m}^{\prime}\left(W_{3}^{\prime}\right) & -U_{2} I_{m}^{\prime}\left(U_{2}\right) & -U_{2} K_{m}^{\prime}\left(U_{2}\right) & 0 \\
0 & 0 & 0 & U_{2}^{\prime} I_{m}^{\prime}\left(U_{2}^{\prime}\right) & U_{2}^{\prime} K_{m}^{\prime}\left(U_{2}^{\prime}\right)-W_{4} K_{m}^{\prime}\left(W_{4}\right)
\end{array}\right]} \\
& \left(n_{3}<n_{\text {eff }}<n_{1}, W I I\right), \\
& =0,
\end{aligned}
$$

where $Y_{m}, K_{m}, J_{m}, I_{m}$ are the Bessel and modified Bessel functions. Dispersion and dispersion slope of monomode fiber are expressed in (17) and (18) respectively [9]:

$$
\begin{aligned}
& D=-\frac{\lambda}{c} \frac{d^{2} n_{4}}{d \lambda^{2}}\left[1+\Delta \frac{d(V B)}{d V}\right]-\frac{n_{4}}{c} \frac{\Delta}{\lambda} V \frac{d^{2}(V B)}{d V^{2}}, \\
& S=-\frac{\lambda}{c} \frac{d^{3} n_{4}}{d \lambda^{3}}\left[1+\Delta \frac{d(V B)}{d V}\right]-\frac{1}{c} \frac{d^{2} n_{4}}{d \lambda^{2}}\left[1+\Delta \frac{d(V B)}{d V}\right]+ \\
& \frac{n_{4}}{c}\left(\frac{\Delta}{\lambda^{2}}\right) V^{2} \frac{d^{3}(V B)}{d V^{3}}+2 \frac{N_{4}}{c}\left(\frac{\Delta}{\lambda^{2}}\right) V \frac{d^{2}(V B)}{d V^{2}}+ \\
& 2 \frac{\Delta}{c} \frac{d^{2} N_{4}}{d \lambda^{2}} V \frac{d^{2}(V B)}{d V^{2}}
\end{aligned}
$$

where $N_{4}=n_{4}-\lambda \frac{d n_{4}}{d \lambda}$, is the group index of outer cladding. $\quad N_{4}$ and $\left(\frac{d n_{4}}{d \lambda}, \frac{d^{2} n_{4}}{d \lambda^{2}}\right)$ represent the material dispersion of pure silica, which can be calculated by the Sellmeier formula [5]. In order to acquire the dispersion and dispersion slope of a mono-mode fiber, it is necessary to compute $\frac{d(V B)}{d V} \cdot V \frac{d^{2}(V B)}{d V^{2}}$ and $V^{2} \frac{d^{3}(V B)}{d V^{3}}$, which represent the waveguide dispersion. Deducing from (7), we obtain [10]:

$$
\frac{d(V B)}{d V}=1+\left(\frac{U_{1}}{V}\right)^{2}\left[1-2\left(\frac{U_{1}}{V}\right) \frac{d U_{1}}{d V}\right],
$$

$$
\begin{aligned}
& V \frac{d^{2}(V B)}{d V^{2}}=-2\left(\frac{d U_{1}}{d V}-\frac{U_{1}}{V}\right)^{2}-2 U_{1} \frac{d^{2} U_{1}}{d V^{2}}, \\
& V^{2} \frac{d^{3}(V B)}{d V^{3}}=-6\left(\frac{d U_{1}}{d V}-\frac{U_{1}}{V}\right)-2 U V_{1} \frac{d^{3} U_{1}}{d V^{3}}- \\
& -6 V \frac{d^{2} U_{1}}{d V^{2}}\left(\frac{d U_{1}}{d V}-\frac{U_{1}}{V}\right), \\
& \text { III. DESIGN AND OPTIMIZING DISPERSION AND } \\
& \text { DISPERSION SLOPE CURVES }
\end{aligned}
$$

\section{DESIGN AND OPTIMIZING DISPERSION AND}

In multi-layer fibers and specially WII fiber, dispersion depends on optical and geometrical parameters. Optimization algorithms are employed to design optical fibers with minimal dispersion and the desired optical and geometrical parameters. In this section, we employ DE optimization algorithm to design a fiber with low dispersion value and flat dispersion slope. A similar approach employing genetic algorithms to improve dispersion can be found in [8]. A DE algorithm cost function (CF) is considered in different ranges of wavelengths. A Gaussian function for the DE algorithm weight function is considered.

$C . F=\sum_{\lambda_{\min }}^{\lambda_{\max }} W(\lambda) \times \mid$ Diapersion $\mid$,

$$
W(\lambda)=\exp \left(\frac{\left(\lambda-\lambda_{0}\right)^{2}}{2 \sigma^{2}}\right)
$$




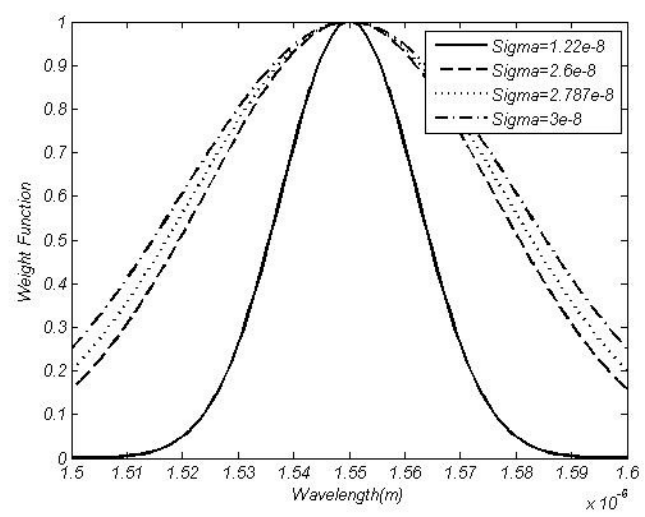

Fig. 2. Gaussian weight function curve applied to the cost function.

Figure 2 represents the Gaussian weight function curve applied to the cost function. Due to the importance of $1.55 \mu \mathrm{m}$ in telecommunications networks, the wavelength interval between $(1.5-1.6 \mu \mathrm{m})$ was chosen, and it was attempted to shift the dispersion at this interval to zero dispersion wavelength.

\section{SIMULATIONS AND RESUlTS}

The dispersion optimization operations are chosen in $(1.5-1.6 \mu \mathrm{m})$ wavelength range. DE algorithm results with $\operatorname{Sigma}(1)=1.22 e-8, \quad \operatorname{Sigma}(2)=1.6 e-8 \quad$ and $\operatorname{Sigma}(3)=2.787 e=8$, with various optical and geometrical parameters are represented in Table 1. The dispersion and dispersion slope curves related to these tables are depicted in Figures 3 and 4 respectively.

TABLE I. GEOMETRICAL AND OPTICAL PARAMETERS OBTAINED FROM THE DE ALGORITHM

\begin{tabular}{|c|c|c|c|}
\hline \multirow{2}{*}{ Parameters } & \multicolumn{3}{|c|}{ Geometrical and optical parameters obtained from the DE algorithm with: } \\
\cline { 2 - 4 } & Sigma(1)=1.22e-8 & Sigma(2)=1.26e-8 & Sigma(3)=2.787e-8 \\
\hline$R_{1}$ & 3.15264670 & 3.09483016 & 3.27005336 \\
\hline$R_{2}$ & -0.92721549 & -0.93197766 & -0.96152876 \\
\hline$a(\mu m)$ & 2.52132231 & 2.51136893 & 2.51245195 \\
\hline$\Delta$ & $55637852 \mathrm{e}-3$ & $54855381 \mathrm{e}-3$ & $53750754 \mathrm{e}-3$ \\
\hline$P$ & 0.76817297 & 0.73308432 & 0.72238260 \\
\hline$Q$ & 0.25397348 & 0.23099590 & 0.22219703 \\
\hline
\end{tabular}

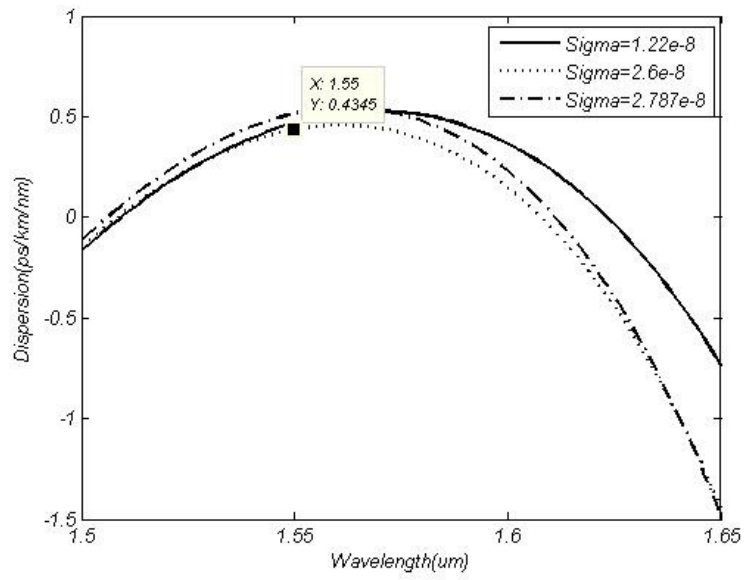

Fig. 3. Dispersion curves in different Sigmas, with shifted optimal wavelength.

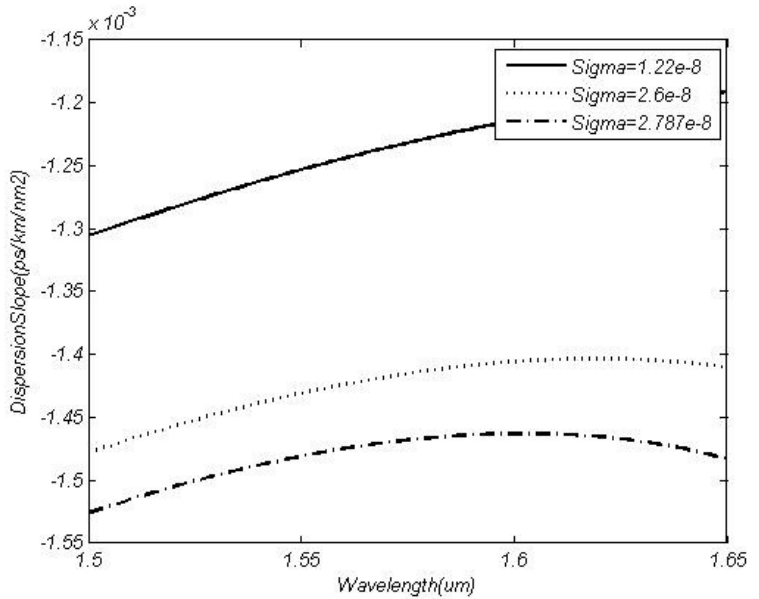

Fig. 4. Dispersion slope curves in different Sigmas in correlation to table(1) 
According to the results and dispersion curves obtained, we can easily see how dispersion wavelength is shifting towards zero dispersion wavelength. Also we can get minimum dispersion with desired sigma value and consequently shift the bandwidth to maximum desired value $(0.1 \mu \mathrm{m})$ in this wavelength range.

Figures 5 and 6 show the dispersion and dispersion slope curves respectively for sigma $=2.6 \mathrm{e}-8$. According to the dispersion slope curve in wavelength range $(1.5-1.6 \mu \mathrm{m})$, we can conclude that in this modeling, dispersion slope is almost flat.

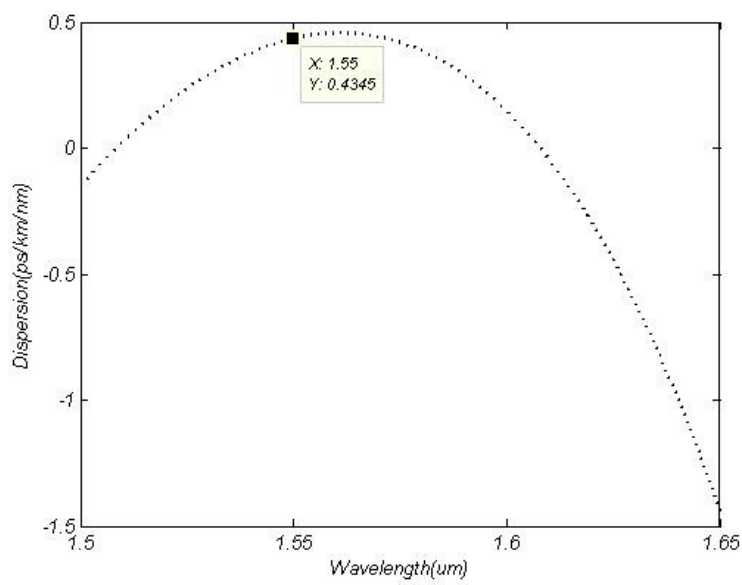

Fig. 5. Dispersion curve in sigma=2.6e-8.

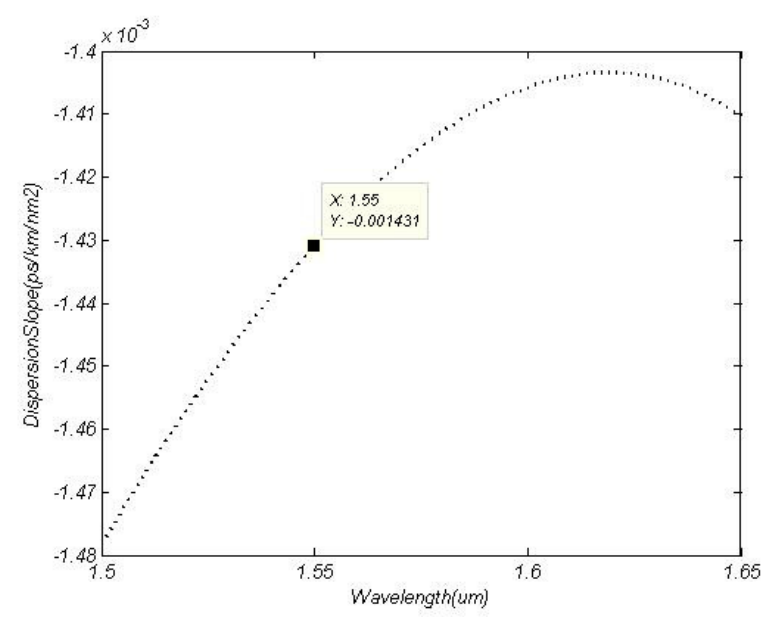

Fig. 6. Dispersion slope curve in sigma $=2.6 \mathrm{e}-8$.

Dispersion value in Figure 5 at $1.55 \mu \mathrm{m}$, equals to $0.4345 \mathrm{ps} / \mathrm{km} / \mathrm{nm}$ which is negligible to the increased bandwidth. It is also shown that the dispersion slope is flat in this bandwidth.

The results obtained here, compared with previous research [7-10] show remarkable improvement both in terms of dispersion and the amount of bandwidth obtained through optimization in DWDM networks.

\section{CONCLUSION}

In this paper, we presented the design of multi-layer optical fibers with wide bandwidth, low dispersion and flat dispersion slope. Calculations show that with increasing distance between two points with zero dispersion, increased dispersion values are achieved. The main purpose of this paper is not to reduce the dispersion dramatically, but to optimize the bandwidth. Therefore, the optimization range of the wavelength is selected so as to increase the bandwidth and not to adversely effect the dispersion. This research, not only provides a novel fiber with flat dispersion, but also gives the basic principle behind the design of any fiber with any desired dispersion curve using appropriate optimization algorithms together with the desired cost function.

\section{ACKNOWLEDGMENT}

This project was financially supported by the Iranian telecommunications research center.

\section{REFERENCES}

[1] Y. Li, C. Hussey, T. Briks, "Triple-clad single-mode fibers for dispersion shifting", Journal of Lightwave Technology, Vol. 11, No. 11, pp. 1812-1819, 1993

[2] P. Tosin, W. Luthe, H. Weber, "Triple-clad fibers for lasers and amplifiers", Conference on Lasers and Electro-Optics EuropeTechnical Digest, p. 44, 1998

[3] H. Hattori, A. Safaai-Jazi, "Fiber designs with significantly reduced nonlinearity for very long distance transmission", Applied Optics, Vol. 37, No. 15, pp. 3190-3197, 1998

[4] J. Cozens, A. Boucouvalas, "Coaxial optical couplers", Electronic Letters, Vol. 18, No. 13, pp. 138-40, 1982

[5] A. Ghatak, K. Thyagarajan, Introduction to fiber optics, Cambridge University Press, 2002

[6] G. P. Agrawal, Fiber-optic communication systems, John Wiley \& Sons. INC. $3^{\text {rd }}$ Ed, 2002

[7] K. Kawano, T. Kitoh, Introduction to optical waveguides analysis, John Wiley \& Sons, 2001

[8] X. Tian, X. Zhang, "Dispersion-flattened design of the large effective-area single-mode fibers with ring index profiles", Optics Communications, Vol. 230, No. 1-3, pp. 105-113, 2004

[9] A. Rostami, S. Makouei, Advances in solid state circuit technologies: a novel multiclad single mode optical fibers for broadband optical networks (chapter 7), ISBN: 978-953-307-086-5, InTech, 2010

[10] M. Savadi-Oskouei, S. Makouei, A. Rostami, Z. D. Koozeh Kanani, "Proposal for Optical fiber designs with Ultrahigh effective area and small bending loss applicable to long haul communications", Applied Optics, Vol. 46, No. 25, pp. 6330-6339, 2007 